\title{
Políticas de información: desarrollo y estrategias de los programas de la UE e impacto en España
}

\author{
María Jesús MARTínez PestañA ${ }^{1}$ \\ Departamento de Biblioteconomía y Documentación \\ Universidad Carlos III de Madrid \\ mjmpesta@bib.uc3m
}

Recibido: Septiembre 2012

Aceptado: Febrero 2013

\begin{abstract}
Resumen: En este estudio se describen las políticas de información procedentes de la Unión Europea y su impacto en el diseño de las políticas de información españolas. Se advierte la escisión entre un modelo regido por la eficiencia y la calidad formulado en la Unión Europea y un contexto económico y social crítico en España desde el año 2010, especialmente en el séptimo Programa Marco vigente hasta el 2013. Por un lado, la Unión Europea ha favorecido el desarrollo de instrumentos específicos relativos a las políticas de información bajo la articulación de programas y proyectos transnacionales, bien como consorcios, Eureka y ESPRIT (European Strategic Programme for Research in Information Technologies), o los sucesivos programas periódicos conocidos como Programas Marco. Ello ha propiciado la creación de organismos e instituciones españolas, como REBIUN (Red de Bibliotecas Universitarias), FECYT (Fundación Española para la Ciencia y la Tecnología) o la Agencia Estatal de Investigación (AEI) de reciente creación (junio 2012). Por otro lado, el diseño de las políticas de información de la Unión Europea señala la aspiración de la Unión Europea a situarse geográfica, económica, tecnológica y estratégicamente frente a otras regiones de peso, como son América del Norte y Japón, como prueba la Agenda Digital 2010, primera iniciativa que ampara el objetivo estratégico Europe 2020.
\end{abstract}

Palabras clave: Política de información; sociedad de la información; estrategia de la Unión Europea

\section{Information policies: development and strategies from EU programmes and their impact in Spain}

\begin{abstract}
This study describes the information policies from the European Union and their impact on the design of Spanish information policies. It is noted the split between a model based on efficiency and quality from the EU, and a critical economic and social context since 2010 in Spain, especially in the seventh Framework Programme lasting until 2013. By one hand, the European Union has encouraged the development of specific instruments concerning information policies, such as various consortia, Eureka and ESPRIT (European Strategic Programme for Research in Information Technologies), and the subsequent periodical programs named Framework Programmes. This frame has facilitated the creation of Spanish
\end{abstract}

${ }^{1}$ Este artículo ha sido elaborado durante la Estancia Postdoctoral en la Universidad Carlos III de Madrid. Facultad de Humanidades, Comunicación y Documentación, Departamento de Biblioteconomía y Documentación, dentro del grupo de investigación ACRÓPOLIS, dirigido por Dra. Mercedes Caridad Sebastián y Dr. Miguel Ángel Marzal García-Quismondo 
institutions as REBIUN (Red de Bibliotecas Universitarias), FECYT (Fundación Española para la Ciencia y la Tecnología), and the recently created Agencia Estatal de Investigación (AEI) (June 2012). By other hand, the information policies scheme from the European Union points out the aspirations of the European Union to stand up geographically, technologically and strategically as a role model compared to other strong regions as North America and Japan, as could be proved by the Agenda Digital 2010, first initiative under the strategic target Europe 2020.

Keywords:Information policy; information society; European Union strategy

\section{INTRODUCCIÓN A LAS POLÍTICAS DE INFORMACIÓN}

Si partimos de la comprensión de lo que significa la Unión Europea, como espacio para el conocimiento, el fortalecimiento económico y que suscribe el principio de subsidiariedad, permitiendo así el desarrollo y participación de las partes (principio de proporcionalidad), resultará sencillo entender las políticas de información emanadas desde la Unión Europea. Recordemos que el principio de subsidiariedad, como reza el art. $5^{\circ}$ del Tratado de la Unión Europea firmado en Maastricht (1992), es un principio organizacional, que entiende la existencia de un centro referente, pero que permite la independencia de las partes.

No se pueden comprender las diferentes políticas de información llevadas a cabo en España sin conocer las políticas aplicadas en forma de programas por la Unión Europea. Aunque se ha cuestionado desde hace años la viabilidad de las mismas debido a las diferencias culturales existentes entre los diferentes países miembros (Brüggemann, 2005; Shore, 1993), no cabe duda que, la aplicación de una serie de herramientas comunes a los diferentes países miembros ha estimulado la formación de modelos o patrones tendentes a desarrollar acciones que con mayor o menor éxito han podido fortalecer las llamadas Tecnologías de la Información y las Comunicaciones (TIC). Sin embargo, estos estímulos son difícilmente sostenibles en un contexto económico y social crítico en España, especialmente, desde el año 2010, como apuntan los indicadores económicos negativos últimos consultados, que aparecen en el primer trimestre del año 2013 (INE).

Si bien los diferentes programas de la Unión Europea son los artífices fundamentales que han contribuido a construir la denominada sociedad de la información, se deben mencionar dos precedentes esenciales que, indudablemente, han contribuido a generar el clima necesario para estimular el desarrollo de herramientas centradas en la calidad y en la eficiencia; se trata de las normas ISO, por un lado, y el fenómeno de la cultura de la calidad en el ámbito de las organizaciones e instituciones, por el otro.

Así, por un lado, las normas ISO (International Organization for Standardization), desarrollas a partir de 1946 y 1947 (Kuert, 1946), han permitido la gestión de la calidad en diferentes ámbitos y actividades económicas (Yeung Mo-ching, 2011; Huei \& Easvaralingam, 2011; Basir, Davies \& Rudder, 2011; Lo, Yeung \& Cheng, 2007; ISO, 2005; Chow-Chua, Goh \& Wan, 2003). A pesar de que las normas ISO 
han podido favorecer el desarrollo de fenómenos de globalización, también han permitido la homologación de normas y pautas de manera preceptiva. Precisamente, en el ámbito de la Tecnologías de la Información y las Comunicaciones, la norma ISO/TC 46 centrada en "información y documentación", ha propiciado la homologación de herramientas para la identificación, indización, clasificación, acceso, selección, explotación, comunicación, intercambio y conservación de información digital y electrónica (ISO, 2010).

Por otro lado, Ansoff y Brandenburg, tras una serie de investigaciones en la década de 1970 relativas a la optimización de la eficiencia en las organizaciones (Ansoff \& Brandenburg, 1969, 1971), señalaron cinco categorías que sentarían las bases de la denominada cultura de la calidad (Sharma, 2005; Meek, 1988); estas categorías que señalaban la eficiencia como patrón de la calidad, evolucionarían posteriormente hacia la gestión de la calidad y la excelencia (EFQM, 2011; Dale et al., 2000). La búsqueda de la eficiencia ha acaparado el interés de muchas investigaciones e indica la necesidad de desarrollar modelos y herramientas que puedan implementar los niveles de calidad y eficiencia y que puedan adaptarse a cualquier tipo de organización (Irani, Beskese \& Love, 2004; Kanji \& Yui, 1997). A partir de 1980 especialmente, se emplea el concepto de cultura de calidad en las organizaciones con vistas a la satisfacción de los consumidores (Hergünern \& Reeves, 2000). Paulatinamente, este concepto se integra en las empresas e instituciones hasta conformar herramientas de gestión no sólo materiales sino humanas y relacionales recorriendo las organizaciones de arriba abajo, dejando de concentrarse en el apartado final, como se había hecho en sus inicios donde importaba más el nivel de satisfacción de los consumidores con un producto, que los mismos procesos susceptibles de ser evaluados y que podrían añadir mejoras en todas las partes del proceso.

La natural evolución hacia la creación de mecanismos que generan normativas (ISO) desde mediados casi del siglo XX, y posteriormente, el desarrollo de herramientas cuya misión ha sido obtener y velar por la eficiencia apenas veinte años más tarde (cultura de la calidad), prologan la exigencia de calidad y eficacia que habrían de ser las señas de identidad de las políticas de información desarrolladas desde la Unión Europea. Tanto es así, que constantemente observamos la elección de los términos calidad, eficiencia y excelencia en las recomendaciones de los programas y proyectos de la Unión Europea.

\section{EL SELLO DE LA UNIÓN EUROPEA}

Los Consejos Europeos de Lisboa en marzo de 2000, Santa María de Feira en junio de 2000 y de Estocolmo en marzo de 2001 aprobaron unas conclusiones dirigidas a la creación rápida de una investigación e innovación de sello europeos, con miras a un crecimiento económico sostenible, más empleo y cohesión social, con el objetivo último de la Unión, para el año 2010 de convertirse en la economía 
del conocimiento más competitiva y dinámica (Kofler, 1998). Basándose en la obligación del artículo 6 del Tratado, el Consejo Europeo de Gotemburgo en junio de 2001, aprobó una estrategia para el desarrollo sostenible y añadió una tercera dimensión ambiental a la estrategia de Lisboa. En particular, el Consejo Europeo de Lisboa subrayó la importancia de la iniciativa e-Europa de la Comisión (Zoltán \& Gábor, 2004), que aspira a una sociedad de la información para todos, mientras que el Consejo Europeo de Estocolmo destacó también la necesidad de hacer un esfuerzo especial en las nuevas tecnologías, especialmente la biotecnología (Matías, 2005).

El Consejo Europeo del año 2000 aprobó en Lisboa su proyecto más ambicioso: convertir Europa en la "sociedad del conocimiento" con más peso económico (Matías, 2005). La fundación de una sociedad del conocimiento no era un proyecto nuevo, pero sí lo fue en su ambiciosa proyección económica, que abarcaba todos los ámbitos económicos y que aspiraba a otorgar a la Unión Europea el liderazgo económico (Zoltán \& Gábor, 2004); la crisis económica que están sufriendo algunos países miembros desde el año 2010 especialmente, ha probado que las diferencias entre los países miembros son más complejas de lo que se estimaba.

Dadas las diferencias e idiosincrasia de los países miembros, con toda seguridad los resultados de las aplicaciones de los programas Marco es muy probable que ofreciesen resultados diferentes en cada uno de los países miembros. De hecho, las críticas más frecuentes se han polarizado entre la institucionalización de una política común investigadora de la Unión y aquellas que prestan atención individualmente a la aplicación de cada uno de los programas en los países integrantes.

Desde una perspectiva crítica, Banchoff adujo que los programas Marco habían configurado la institucionalización de las políticas de investigación y que en realidad, el objetivo último era la creación de un área de investigación europea, articulada para competir con la superioridad tecnológica y científica de Estados Unidos (Goodwin \& Spittle, 2002). Pero que, a pesar de los esfuerzos de coordinación entre los países miembros, persistían diferentes políticas nacionales entre los países miembros (Banchoff, 2002). Asimismo, otros autores (Arnold, Clark \& Muscio, 2005) con posteridad estimaron que, a pesar de la continua evaluación a la que están sujetos los programas Marco y los claros beneficios para los países participantes, era necesaria una actitud sistemática de evaluación, así como el uso de la inteligencia estratégica por parte de la Comisión Europea. Se subrayó además que las evaluaciones pueden ser problemáticas ya que, en palabras de Alesina y Perotti, existe en sí un conflicto entre las posiciones de los diferentes gobiernos miembros con posturas manifiestas más libres de mercado, o dirigidas, como es el caso de los países del área mediterránea, entre otros (Alesina\&Perotti, 2004: 6-7). 


\section{EUREKA, ESPRIT Y LOS PROGRAMAS MARCO}

Las políticas de información, como conjunto de directrices impulsadas desde la Unión Europea han mantenido una singladura estratégica definida como se puede observar en Europe 2020.

De hecho, se considera que la Unión Europea inició formalmente "su estrategia política en el sector de la sociedad de la información" en la década de 1980. Precisamente ESPRIT (en Tecnologías de la Información) y el programa RACE (Tecnologías de Telecomunicaciones Avanzadas) agruparon las primeras actividades en el campo de las TIC (Tecnologías de la Información y las Telecomunicaciones). Además el Libro Verde (1987) dio lugar a la liberalización de las telecomunicaciones (FECYT). A partir de este momento, todos los programas de importancia, desde El Libro Blanco (1993) hasta Europe 2020 integran las TIC como objetivos esenciales para crear en Europa la sociedad de la información y así convertir Europa en la economía basada en el conocimiento más competitiva (FECYT).

Desde el momento en que se lanza la iniciativa Eureka, creada durante la Conferencia de París sobre tecnología europea en 1985 por 19 países a los que se añadirían los países de la Comunidad Europea, la Comisión Europea contempló esta iniciativa como complementaria a sus propias actividades de investigación y desarrollo tecnológico. De hecho, la Comisión Europea insistiría en completar los proyectos de Eureka con los propios programas de la Unión para así asegurar la continuidad de los mismos (EU, 1988a, 1994a, 1994b) y también evitar duplicidades (EU, 1988b). Baste un ejemplo: en 1994 la Unión participaba ya en 27 de los proyectos de iniciativa Eureka, convertida ya en "protagonista principal de la cooperación tecnológica europea" (EU, 1994a).

La clave del éxito de Eureka reside en su orientación práctica de proyectos de I+D en el mundo empresarial e industrial, centros de investigación y universidades. La flexibilidad a la hora de buscar financiación tanto privada como gubernamental para el desarrollo de los mismos, y la descentralización, ha permitido que la iniciativa Eureka haya podido participar en diferentes programas Marco (EU, 1988a).

Tanto los consorcios de Eureka como los de ESPRIT (European Strategic Programme for Research in Information Technologies) poseen un carácter transnacional; sin embargo, Eureka, aunque orientado a las tecnologías en general, carece de limitación en cuanto a sectores, ya que los proyectos están cercanos a los mercados y los participantes tienen libre elección en cuanto a los temas de investigación, así como tienen libertad para localizar financiación privada o pública entre los gobiernos participantes. Es más, algunos proyectos poseen doble adscripción (Eureka y ESPRIT), es decir, proyectos cofinanciados por la Comisión y con créditos del programa ESPRIT (EU, 1994b: 12).

Como ha quedado definido en el Diario Oficial, los programas ESPRIT "son programas específicos de investigación de gastos compartidos que corresponden a 
sucesivos programas Marco"; y lo más importante, "[t]ienen por objeto la tecnología de la información (TI)", es decir, técnicas propias de la microelectrónica, de equipos informáticos y software (EU, 1994b: 3). Los programas ESPRIT constituyen además, un instrumento estratégico (EU, 1994a) que promueve la cooperación y participación entre industrias, universidades y centros de investigación.

El espíritu de I+D que promueve la Unión Europea a través de los diferentes programas Marco, facilitará la integración de ESPRIT en diferentes programas Marco, desde que fuese aprobado en 1984 en el Consejo de La Unión Europea (EU, 1988c). A partir de esta fecha, en la que 1983 y 1984 se consolidaron como fase preparatoria de "un programa comunitario de investigación y desarrollo en el ámbito de las tecnologías de la información", ESPRIT se integraría en los diferentes programas Marco. Así, se menciona ESPRIT I, correspondiente al primer Programa Marco (19841988) titulado "Programa europeo de investigación y de desarrollo en el ámbito de las tecnologías de la información". ESPRIT II, "Programa europeo estratégico de investigación y de desarrollo en el ámbito de las tecnologías de la información" correspondiente al segundo Programa Marco (1987-1991). Adscrito al tercer Programa Marco (1990-1994), se desarrolla el "Programa específico de investigación y de desarrollo en el ámbito de la tecnología de la información (1990-1994)" (1991-1994) (EU, 1988c, 1994a; EU, 1994b: 5; EU, 1997).

Si bien los tres primeros programas Marco contaban con presupuestos que no sobrepasaban los 7 billones de euros, a partir del cuarto Programa Marco (19941998) se duplican; y de hecho, las cifras son similares tanto para el quinto (19982002) como para el sexto Programa Marco (2002-2006). El quinto Programa Marco, que sustituyó al anterior, comprendía a su vez seis programas, fruto de las seis prioridades identificadas en la comunicación Inventing Tomorrow; la segunda de las prioridades hacía referencia expresa a la creación de una sociedad de la información accesible ("user-friendly information society") (EU, 1996).

Debemos anotar que hasta el sexto Programa Marco, los períodos para cada programa abarcaban períodos no superiores a los 5 años, a partir del séptimo Programa Marco se extiende la duración a 7 años y se multiplican los presupuestos. De hecho, el octavo Programa Marco está ya aprobado con un presupuesto estimado de 80.000 millones de euros y abarcará desde los años 2014-2020.

El actual séptimo Programa Marco de Investigación y Desarrollo Tecnológico (2007-2013) prueba en su presupuesto superior a los 50.000 millones de euros que la investigación en Europa es una prioridad. El gran grueso de esta cantidad se ha invertido en la cofinanciación de proyectos de investigación para el desarrollo tecnológico y proyectos con base práctica (demostraciones) fundamentalmente. Los diferentes programas Marco han trabajado en la posición estratégica de la investigación europea a nivel mundial; ello también es fundamental en el séptimo Programa Marco que incide en la transcendencia más allá de fronteras nacionales y la cooperación entre países (EC, 2007). 


\section{CALIDAD Y EXCELENCIA EN LA INVESTIGACIÓN UNIVERSI- TARIA ESPAÑOLA}

Una importante fuente de financiación de la actividad investigadora de la universidad española ha venido de la mano de los programas Marco, puesto que la participación de España en los programas Marco se inicia ya en 1986. La financiación ha permitido también la colaboración entre diferentes universidades, empresas europeas y centros de investigación (CRUE, 2002: 11).

La Conferencia de Rectores de Universidades Españolas (CRUE) ya declaró que el sexto Programa Marco era un instrumento fundamental para el desarrollo de políticas científicas y tecnológicas dentro de la Unión Europea; en especial porque en este programa "se definen las líneas de investigación y desarrollo tecnológico que financia la UE y se fijan las dotaciones presupuestarias" (CRUE, 2002: 11). Estas dotaciones no tenían otro objetivo más que fortalecer las bases científicas y tecnológicas dentro de la Unión Europea. Ya que, a pesar de ser conocido el impacto negativo debido a un uso deficiente de los mecanismos de control (Lee, Khong \& Guista, 2006; Yorke, 2000), también es verdad que no se integraría hasta muy tardíamente la valoración de los resultados estrictamente técnico-científicos (EU, 1994: 5).

A pesar de que no hayan existido mecanismos de control rigurosos, la Unión Europea ha propiciado, sin embargo, la creación de una serie de organismos para la evaluación de la calidad en el marco de las universidades, especialmente a raíz del llamado "proceso de Bolonia" (ENQA, 2009). En el caso de España, contamos con dos instituciones fundamentales para la evaluación en el ámbito de la universidad: CRUE y ANECA. En 1988, se creó REBIUN (Red de Bibliotecas Universitarias) a iniciativa de las universidades españolas, cuya finalidad inicial fue fomentar la cooperación entre las universidades españolas; posteriormente se incorporaría como una de las comisiones sectoriales en CRUE (REBIUN). Ya en 1994 se constituye la Conferencia de Rectores de las Universidades Españolas, CRUE; a este organismo están asociadas casi la totalidad de las universidades españolas públicas y privadas. La labor de CRUE se centra principalmente en el fomento del la educación e investigación universitaria tanto a nivel nacional, como en cooperación con universidades extranjeras (REBIUN).

Precisamente, siguiendo el hilo conductor del estímulo investigador fomentado desde la Unión Europea, se crea en España la Fundación Española para la Ciencia y la Tecnología (FECYT). Dependiente del Ministerio de Economía y Competitividad, FECYT pretende impulsar, desde su creación en 2001, la ciencia y la innovación tecnológica, marcando la apertura al mundo empresarial. De hecho, su objetivo clave es "contribuir al desarrollo de una economía basada en el conocimiento" (FECYT).

Al amparo de un contexto general europeo impulsor del fomento de la búsqueda de la calidad y la coordinación para la mejora de la educación e investigación 
universitaria, se crea la Agencia Nacional para la Evaluación de la Calidad y Acreditación (ANECA) en el año 2002. Esta agencia española, no sólo tiene como misión potenciar la actividad docente e investigadora de la universidad española, sino que también, como gestora del rendimiento de la educación universitaria en nuestro país, se ha convertido en árbitro asesor para el cumplimiento de planes y proyectos, tanto nacionales como internacionales.

A estas instituciones españolas, se añade la Agencia Estatal de Investigación (AEI), creada en junio de 2012 con el objetivo de "crear instrumentos y mecanismos de financiación y gestión más eficientes que mejoren el impacto de la inversión de la Administración central en investigación" (MINECO), pero que no se espera que esté en funcionamiento hasta avanzado el 2013. Esta Agencia se añade a otras instituciones públicas o dependientes de la Secretaría de Estado de Investigación, Desarrollo e Innovación, como el Consejo Superior de Investigaciones Científicas (CSIC), el Instituto Geológico y Minero de España (IGME), el Instituto Nacional de Investigación y Tecnología Agraria y Alimentaria (INIA), el Instituto de Salud Carlos III (ISCIII), el Museo Nacional de Ciencia y Tecnología (MUNCYT), el Centro de Investigaciones, Energéticas, Medioambientales y Tecnológicas (CIEMAT), el Instituto Español de Oceanografía (IEO), el Instituto de Astrofísica de Canarias (IAC), la mencionada Fundación Española para la Ciencia y Tecnología, el Centro para el Desarrollo Tecnológico Industrial (CDTI) y la Fundación Genoma España.

Las instituciones arriba mencionadas son dependientes de la Secretaría de Estado de Investigación, Desarrollo e Innovación. Se debe aclarar que esta Secretaría de Estado asume, tanto las competencias de su ámbito (investigación científica y técnica, desarrollo e innovación), como también dirige y coordina las relaciones internacionales y de la Unión Europea.

El motor de la Unión Europea, mediante el desarrollo de programas y la creación de consorcios con espíritu transnacional, ha sido fundamental para la creación de agentes nacionales impulsores del desarrollo e investigación científica, tanto empresarial como en el marco de las universidades en España. A pesar de las críticas o deficiencias que puedan existir, no dejan de ser un estímulo para implementar acciones y resultados; y no se pone ya en cuestión el impulso tan importante de la Unión Europea y, en especial, el papel de los programas Marco como motor para la investigación. De hecho, que España sea país coordinador de 5.816 proyectos con el sello de Programa Marco a fecha de junio de 2012, revela el efecto impulsor de la Unión Europea como aspirante a convertirse en un espacio para la creación de conocimiento e investigación. Baste anotar, que por encima de España como país coordinador de proyectos en el año 2012, figuraba en cabeza Reino Unido con 17.357 proyectos, seguido de Alemania con 13.191, Francia con 12.464, Italia con 8.402 y Holanda con 6.371 proyectos (CORDIS). Aunque se desconocen los resultados exactos de todos los proyectos financiados, dados los problemas administrativos y la falta de mecanismos de evaluación en muchos ca- 
sos, muchos resultados de algunos proyectos en áreas de biología, medicina, medioambiente, tecnologías y transportes aparecen publicados mensualmente en $R e$ search*EU Results (CORDIS).

Sin embargo, este escenario positivo arriba mencionado contrasta dramáticamente con la situación económica española en el primer trimestre del año 2013, como reflejan los últimos datos (INE), que manifiestan una situación económica crítica (crecimiento galopante del desempleo, descenso importante del consumo familiar y empresarial, descenso del PIB, entre otros indicadores), agravada con un importante problema social, como evidencian las cifras de emigración de población juvenil a Europa.

\section{EL SIGNIFICADO DE EUROPE 2020}

Existe un antes y un después en los programas y proyectos impulsados desde la Unión Europea desde las primeras iniciativas hasta el año 2012. Si anteriormente, se habían fomentado proyectos y programas sin compromisos en los resultados, como se dijo en uno de los últimos programas Marco, y sin una estrategia expresa, Europe 2020 constituye una propuesta mucho más arriesgada.

A caballo del fin del séptimo y el inicio del octavo Programa Marco, Europe 2020 tiene unas marcadas líneas de actuación, sino más ambiciosas, sí más críticas con empresas y gobiernos y más claras en sus objetivos concretos. El objetivo del 2020 está articulado en un tripe crecimiento: el inteligente (educación, investigación e innovación), el sostenible (medio ambiente) e integrador (social). A este tripe crecimiento, se añaden cinco objetivos importantes que, sin ser del todo novedosos, lo son las acciones a tomar, como son: empleo, I+D, cambio climático y energía, educación y lucha contra la pobreza y la exclusión social (EC).

La misma Comisión declaró en el año 2010 que había ideado un plan para "esbozar la prosperidad y el bienestar europeos" (EU, 2010); era la Agenda Digital. Este plan no era una mera propuesta sobre las TIC, sino que insertaba claramente contenidos económicos. De hecho, la Agenda Digital 2012 corresponde a una de las primeras acciones de Europe 2020. Tal vez sean aclaratorias las palabras de Neelie Kroes, Vicepresidenta de la Comisión y responsable de la Agenda Digital, quien expresaba dos años más tarde que se deben "situar los intereses de los ciudadanos y las empresas europeos entre las prioridades de la revolución digital y, de esta manera, maximizar el potencial de las tecnologías de la información y las comunicaciones (TIC) para incrementar la creación de empleo, la sostenibilidad y la inclusión social». Además, el responsable de la Agenda Digital afirmaba que "[1]a ambiciosa estrategia presentada hoy señala claramente hacia dónde tenemos que dirigir nuestros esfuerzos en los próximos años. Para materializar plenamente el potencial del futuro digital de Europa, debemos contar con el compromiso incondicional de los Estados miembros, el sector de las TIC y otros agentes económicos de importancia vital" (EU, 2012). 
Los objetivos de la Agenda Digital son de aplicación nacional. España, a través del Observatorio Nacional de las Telecomunicaciones (ONTSI), dependiente del Ministerio de Industria, Energía y Turismo, ha publicado los principales indicadores del sector TIC, de la sociedad de la información y de la Agenda Digital para Europa 2020 necesarios para cumplir los objetivos. Aunque los datos pertenecen al año 2010 y 2011, los objetivos para el año 2015 son definitorios y se refieren a la Banda Ancha, a usos de Internet, a la Administración electrónica (e-Government) y al Comercio electrónico (e-Commerce).

Las recomendaciones del Grupo de Expertos para la Agenda Digital para España, constituido en marzo de 2012, habían señalado seis objetivos que, contribuirían a mejorar la competitividad empresarial española, a general empleo de mayor calidad y unos servicios públicos más eficientes (Pérez, 2012). De hecho, el Informe de recomendaciones del Grupo de Expertos de alto nivel para la Agenda Digital para España, publicado en junio de 2012, estimaba que "la adopción inteligente de tecnologías digitales permitirá impulsar el crecimiento, la innovación y la productividad, contribuyendo a evitar que se trunque la trayectoria de transformación y modernización que ha experimentado la economía española en las últimas décadas" (Pérez, 2012: 9). Además se señalaba que impactará y beneficiará tanto a la sociedad como a la economía españolas. Ya que, añaden, facilitará la creación de nuevos negocios, transformará la mediana empresa y la hará más competitiva y modificará los procesos productivos. Además, insisten en que "[1]a necesaria metamorfosis de nuestra economía para hacerla más eficiente, innovadora, competitiva e internacionalizada, sobre la base de las Tecnologías de la Información y la Comunicación, puede y debe ser impulsada mediante una estrategia nacional" (Pérez, 2012: 10)". Este mismo informe anotaba seis objetivos principales que conformaban la estructura de la Agenda Digital:

1. fomentar el despliegue de servicios y redes que garanticen la conexión digital;

2. impulsar la economía digital en la empresa española, para mejorar el crecimiento, competitividad e internacionalización;

3. mejorar los servicios públicos (e-Administración) y las soluciones digitales de los mismos;

4. que el ámbito digital goce de garantías para la privacidad, la seguridad y la confianza;

5. que se impulsen la investigación, el desarrollo y la innovación $(\mathrm{I}+\mathrm{D}+\mathrm{i})$ en el ámbito de las Tecnologías de la Información y las Comunicaciones (TIC);

6. y, finalmente, que se promuevan la formación de nuevos profesionales TIC, así como la capacitación para la inclusión digital. 
Sin embargo, los objetivos arriba mencionados se sitúan, a fecha de 2013, en un conjunto de buenas intenciones. De acuerdo con datos publicados en el Informe eEspaña 2012 editado por la Fundación Orange (Gimeno, 2012: 190), "España se encuentra en la decimoséptima posición en términos de desarrollo de la SI [Sociedad de Información] en Europa y en la decimocuarta en términos de riqueza de sus habitantes". Las perspectivas no son más halagüeñas para el año 2013 donde se ha reducido, entre otras partidas, la inversión en I+D; solo basta consultar los indicadores económicos y financieros presentes en el INE a principios del año 2013, entre los que abundan los datos negativos en comparación incluso con el año 2012 (INE, 2013).

Los últimos informes, tanto del Observatorio Nacional de las Telecomunicaciones y de la Sociedad de la Información, como el de la Fundación Telefónica y de la Fundación Orange (ONTSI, 2012; Fundación Telefónica, 2012; Gimeno, 2012), coinciden que tanto el comercio electrónico (e-Commerce) como la Administración electrónica (e-Government) son los que necesitan crecer para el año 2015, ya que están por debajo de la media de la Unión Europea; mientras que la penetración de la Banda Ancha o el número de particulares que usan habitualmente Internet están más cercanos a la media europea. Además, de acuerdo con el mencionado informe publicado por Fundación Orange, si bien la Agenda Digital juega un importante papel en la estrategia Europe 2020, la contracción económica que sufre España, a pesar del millón de nuevos internautas, ha propiciado que la economía digital se haya contraído de manera apreciable en España (Gimeno, 2012: 190); y ello queda demostrado en una caída superior al 10\% en las importaciones del sector TIC. También, se debe añadir que el cambio de gobierno en 2011 ha retrasado, no solo la puesta en marcha de la Agenda Digital en España, sino que ha dejado en suspenso Estrategia Digital (2011-2015) (Gimeno, 2012: 190). De hecho, si bien España había acortado diferencias con Europa durante el período 2006-2009, se produce una tendencia contraria en el 2010, confirmada en el 2011, situando el desarrollo de la sociedad de la información en España en dos puntos por debajo de la media europea y que sitúa a España "en una posición similar a la del año 2008" (Gimeno, 2012: 191). A ello, se suma el riesgo de exclusión digital por parte de las mujeres de mayor edad, amas de casa y desempleados, como advierte el referido informe.

Sin duda, las acciones que enmarcan la Agenda Digital en España con objetivos para el año 2013 y 2015, componen parte de una arquitectura más grande y ambiciosa; donde si bien las Tecnologías de la Información y Telecomunicaciones, son parte fundamental, no es menor el acento que se pone en el crecimiento económico de España y, por extensión, el de la Unión Europea, al ser coincidentes los objetivos.

Aunque en marcha, la Agenda Digital es el primer plan que forma parte de esa arquitectura estratégica señalada hasta el año 2020. Resta por ver los resultados finales de ese primer plan y los sucesivos dentro de Europe 2020. Sin embargo, no 
se puede ya ignorar que la Unión Europea y los países miembros enmarcan un área que aspira a ser centro estratégico, capaz de reportar beneficios económicos y tecnológicos, más allá del mapa del conocimiento.

\section{CONCLUSIONES}

La creación de un espacio europeo impulsor del conocimiento y la investigación, no podría tener lugar sin el desarrollo de una serie de políticas de información concretas. Con anterioridad, la eficiencia y la búsqueda de la calidad, desde el ámbito organizacional, o desde instituciones como ISO, constituyen prólogos esenciales, puesto que señalan el fomento y la gestión de la calidad como agentes esenciales que habrían de implantarse en los diferentes programas y proyectos europeos. Eureka y ESPRIT, instituciones creadas para el desarrollo científico y tecnológico son parte actante y motor fundamental de la aspiración de la Unión Europa a convertirse en espacio del conocimiento con peso específico frente a otras áreas.

La trayectoria hacia la creación de un espacio europeo estratégico y competitivo, el desarrollo y articulación de programas y su implantación en los diferentes espacios, en concreto en España, ha favorecido el crecimiento y desarrollo no sólo de proyectos sino también la creación de agentes esenciales en el ámbito del desarrollo científico e investigador como son REBIUN, FECYT y ANECA. Estos agentes, a los que se suma la Agencia Estatal de Investigación, tienen una característica común, la búsqueda de la eficiencia y la calidad. Mediante diferentes iniciativas auspiciadas desde la Unión Europea, España se ha incorporado a iniciativas que propiciarían el intercambio de personas y conocimiento entre instituciones y países.

Es más, la implicación de España en el trasvase de conocimiento mediante la participación en proyectos promovidos desde la Unión Europea, es revelador. España es país coordinador de más de 5.800 proyectos de algún Programa Marco, y ocupa la sexta posición como país.

En definitiva y sin que sea novedoso, la puesta en marcha de Europe 2020 prueba la aspiración expresa de la Unión Europea a convertirse en área estratégica en el área de las tecnologías y el conocimiento frente a otras áreas geográficas como América del Norte y Japón. La puesta en marcha de la Agenda Digital en la Unión Europea, así lo confirma.

Sin embargo, las aspiraciones arriba señaladas, contrastan con el panorama en España, donde se ha producido el retraso en la puesta en marcha de la Agenda Digital, el retroceso en el avance del desarrollo de la sociedad de la información, situada ya en posiciones equivalentes a las de 2008, además de un contexto económico y social crítico en todo el país. Este escenario particular obliga a cuestionar la viabilidad de las aspiraciones de Europe 2020, al menos en el conjunto de la UE. 


\section{REFERENCIAS BIBLIOGRÁFICAS}

ALESINA, Alberto; Perotti, Roberto (2004). The European Union: A Politically Incorrect View. Journal of Economic Perspectives, 18(4), 27-48. Disponible en: http://www.nber.org/ papers/w10342. (Consulta: 24/05/2012)

ANECA, Agencia Nacional de Evaluación de la Calidad y Acreditación. En: http://www.aneca.es/.

ANSOFF, H.I.; BRANDENBURG, R.G. (1971). A language for organization design: Part II. Management Science, 17(12), B717-B731. Disponible en: http://www.jstor.org/stable/ 2629463 (Consulta: 12.04.2012).

ANSOFF, H.I.; BRANDENBURG, R.G. (1969). The General Manager of the future. California Management Review, 11, 61-72. Disponible en: $\mathrm{http} / / /$ search.proquest.com/docview/206285046/fulltextPDF?accountid=14501. (Consulta: 12/04.2012).

ARNOLD, E.; CLARK, J.; MUSCIO, A. (2005). What the evaluation record tells us about European Union Framework programme performance. Science and Public Policy, 32(5), 385-397. DOI: 10.3152/147154305781779335.

BANCHOFF, T. (2002). Institutions, inertia and European Union research policy. JCMS: Journal of Common Market Studies, 40(1), 1-21. DOI: 10.1111/14685965.00341.

BASIR, SITI A.; DAVIES, JOHN; RUDDER, ALLISON (2011). African Journal of Business Management, 5 (15), 6028-6035. Disponible en: Available online at http://www.academicjournals.org/AJBM (Consulta: 22.03.2012).

BRÜGGEMANN, Michael (2005). How the EU constructs the European public sphere: Seven strategies of information policy. TranState Working Papers, 19. Disponible en: http://hdl.handle.net/10419/28269 (Consulta: 23.03.2012).

CHOW-CHUA, Clare; Goh, Mark; Wan, Tan Boon (2003). New Research: Does ISO 9000 certification improve business permormance? International Journal of Quality \& Reliability Management, 20(8), 936-953. DOI 10.1108/ 026567 10310493643.

CORDIS, Community Research and Development Information Service. En: http://cordis.europa.eu/projects/index.cfm?fuseaction=app.search\&T. (Consulta: $07 / 06 / 2012$ )

CRUE, Conferencia de Rectores de las Universidades Españolas (2002). Análisis de la participación e impacto del programa marco de investigación y desarrollo tecnológico de la UE en las universidades españolas. Recomendaciones para el futuro .Disponible en: http://crue.org/export/sites/Crue/ Publicaciones/ Documentos/Otras_publicaciones/Univ_Programa_Marco_UE.pdf. (Consulta: 02.08.2012)

CRUE, Conferencia de Rectores de las Universidades Españolas. En: http://www.crue.org/ 
DALE, B.G.; et al. (2000). Quality is dead in Europe - long live excellence - true or false? Measuring Business Excellence, 4(3), 4-10. Disponible en: http://search.proquest.com/docview/208744144? accountid=14501 (Consulta: 10.03.2012).

EC, European Commission (2007), FP7 in Brief. How to get involved int EU 7th Framework Programme for Research. Luxembourg, Office for Oficial Publications of the European Communities. ISBN 92-79-04805-0. Disponible en: http://ec.europa.eu/research/fp7/pdf/fp7-inbrief_en.pdf(Consulta: 22.03.2012).

EC, European Commission, Europe 2020. En: http://ec.europa.eu/europe20120/

EFQM, European Foundation for Quality Management (2011). EFQM Annual Report 2010-2011. Disponible en: http://www.efqm.org/en/PdfResources/EFQM\% 20Annual\%20 Report\%202010.pdf (Consulta: 2402 2012).

ENQA (2009). Standards and Guidelines for Quality Assurance in the European Higher Education Area. Helsinki, European Association for Quality Assurance in Higher Education. Disponible en: http://www.enqa.eu/pubs.lasso. (Consulta: 21.05.2012)

EU (2012). Agenda Digital: El marcador anual confirma la necesidad de reformas económicas estructurales en toda Europa, un excedente de empleo en el sector de las TIC y una fuerte tendencia hacia la tecnología y los servicios móviles (Comunicado de prensa). Referencia: IP/12/614, fecha 18 de junio, 2012.

EU (2010). Agenda Digital: La Comisión esboza un plan de acción de acción para esbozar la prosperidad y el bienestar europeos (Press release). Reference: IP/10/581. Fecha: 19 de mayo, 2010. Disponible en: http://europa.eu/rapid/pressReleasesAction.do?reference=IP $/ 10 / 581 \&$ format $=$ HTML\&aged $=0$ \&language $=$ ES\& guiLanguage $=$ es.$($ Consulta: 28.06.2012)

EU (1997). Welcome to Esprit, the information technologies programme, Cordis Archive. Disponible en: http://cordis.europa.eu/esprit/src/intro.htm. (Consulta: 23.05. 2012)

EU (1996). The Commission clarifies its intentions concerning the Fifth Researchframework programme (Press release). Reference IP/96/1052, date 20/11/1996. Disponible en: http://europa.eu/rapid/press Releases Action.do?reference $=\mathrm{IP} / 96 / 1052 \&$ format=HTML. (Consulta: 23.05. 2012)

EU (1994a). Commisioner Ruberti at the Eureka Ministerial Conference, Lillehammer, 16 June (Press release). Reference IP/94/545, date 16/06/1994. Disponible en: http://europa.eu/rapid/pressReleases Action.do?reference $=I P / 94 / 545 \&$ format=HTML. (Consulta: 21.05 .2012$)$

EU (1994b). "Informe especial n ${ }^{\circ} 6 / 93$ sobre los programas europeos de investigación y de desarrollo en el ámbito de la tecnología de la información (programas ESPRIT) acompañado de las respuestas de la Comisión”. Diario Oficial de las Comunidades Europeas del 14 de febrero de 1994, C 45, año 37. 
EU (1988a). The Commission is willing to reinforce the cooperation with Eureka, 22 June (Press release). Reference IP/88/385, date 22/06/1988. Disponible en: $\mathrm{http} / /$ europa.eu/rapid/pressReleasesAction.do?reference $=\mathrm{IP} / 88 / 385 \&$ format $=\mathrm{H}$ TML\&aged $=1 \&$ language $=E N \&$ guiLanguage $=$ en. $($ Consulta: 21.05 .2012$)$

EU (1988b). Significant results of the Research Council (Press release). Reference MEMO/88/55, date 12/04/1988. Disponible en: http://europa.eu/rapid/ pressReleasesAc-

tion.do?reference $=\mathrm{IP} / 88 / 385 \&$ format $=$ HTML\&aged $=1$ \&language $=\mathrm{EN} \&$ guiLan guage $=$ en. (Consulta: 23.05.2012)

EU (1988c). "Decisión del Consejo de 11 de abril de 1988 relativa al programa estratégico europeo de investigación y de desarrollo en el ámbito de las tecnologías de la información (ESPRIT). Diario Oficial de las Comunidades Europeas. L 118/32. Disponible en: http://eur-lex.europa.eu/LexUriServ/ LexUriServ.do?uri=OJ:L:1988:118:0032:0041:ES:PDF (Consulta: 04/06/20 12)

FECYT, Fundación Española para la Ciencia y la Tecnología. En: http://www.fecyt.es/.

Fundación Telefónica (2012). La sociedad de la información en España 2011. Madrid: Ariel, Fundación Telefónica. ISBN: 978-84-08-00383-0.

GIMENO, M. (Dir.) (2012). Informe eEspaña 2012. Madrid: Fundación Orange. Disponible en: http://fundacionorange.es/fundacionorange/analisis/eespana/e_espana12.html (Consulta: 19.02.2013)

GOODWIN, Ian; SPITTLE, Steve (2002). The European Union and the Information society. New Media \& Society, 4(2), 225-249. DOI: 10.1177/14614448020 0400206.

HERGÜNERN Gulten; REEVES, N.B.R. (2000). Going against the national culture grain: A longitudinal case study of organizational culture. Total Quality Managemnet \& Business Excellence, 11(1), 45-56. Disponible en: http://search.proquest.com/docview/219802249/ fulltextPDF?accountid=14501. (Consulta:10.04.2012).

HUEI, Cham Tat; Easvaralingam, Easvaralingam (2011). Perceptions of Service Quality, Corporate Image, and Customer Loyality in the Hotel Industry of Malaysia. The 2nd International Research Symposium in Service Management. Yogyakarta, INDONESIA, 26 - 30 July 2011. Disponible en: http://irssm.upnyk.ac.id/userfiles/file/papers/016.pdf (Consulta: 20.03. 2012).

INE (2013). España: Indicadores económicos y financieros. Disponible en: http://www.ine.es/fmiFrontEnd/fmi.jsp?L=0 (Consulta: 20.02.2013).

IRANI, Z.; BESKESE, A.; LOVE, P.E.D. (2004). Total quality management and corporate cultura: Constructs of orgnanisational excellence. Technovation, 24, 643-650. DOI: $10.1016 / \mathrm{S} 0166-4972(02) 00128-1$.

ISO (2010). ISO/TC 46 Business Plan 2010. Version N2258. n2258_TC46_Business Plan2010.doc. Disponible en: 
http://isotc.iso.org/livelink/livelink/fetch/ 2000/2122/687806/ISO_TC_046_Information_and_documentation_.pdf?node id $=1959966 \&$ vernum $=-2$. (Consulta:19.03.2012).

ISO (2005). The ISO Survey of Certifications. Disponible en: http://www.iso.org /iso/survey2005.pdf (Consulta:19.03.2012).

KANJI, Gopal K; Yui, Hiroshi (1997). Total quality culture. Total Quality Management \& Business Excellence, 8 (6), 417-428. Disponible en: http://search. proquest.com/docview/219817368 (25.03.2012).

KOFLER, Angelika (1998). Digital Europe 1998: Policies, technological development and implementation of the emerging Information Society. Innovation: The European Journal of Social Sciences, 11(1), 53-71. DOI: 10.1080/13511 610.1998 .9968551$.

KUERT, Willy (1946). The Founding of ISO. Swiss delegate to the London Conference 1946. Disponible en: http://www.iso.org/iso/founding.pdf (Consulta: 23.03. 2012).

LEE, Pui-Mun; Khong, Poh W.; Ghista, Dhanjoo N. (2006). Impact of deficient healthcare service quality. The TQM Magazine, 18(6), 563-571. Disponible en: http://dx.doi.org/10.1108/09544780610707075 (Consulta: 10.04.2012)

LO, Chris K.Y.; Yeung, Andy C.L.; Cheng, T.C. Edwin (2007). Impact of ISO 9000 on time-based performance: An event study. World Academy of Science, Engineering and Technology, 30(7), 35-40. Disponible en: http://www .waset.org/journals/waset/v30/v30-7.pdf. (Consulta: 21.03.2012).

MATÍAS Clavero, G. (2005). La estrategia de Lisboa sobre la sociedad del conocimiento: La nueva economía. ICE, 820, 169-193.

MEEK, V.L. (1988). Organizational culture: Origins and weakness. Organization Studies, 9, 453-473. DOI: 10.1177/017084068800900401.

MINECO, Ministerio de Economía y Competitividad. En: http://www.mineco.gob.es/

ONTSI, Observatorio Nacional de las Telecomunicaciones y de la Sociedad de la Información (2012). Indicadores destacados de la sociedad de la información. Folleto. Disponible en: http://www.ontsi.red.es/ontsi/sites/ default/files/indicadores_destacados_si_junio_2012.pdf. (Consulta: 20.06. 2012)

PÉREZ MARTÍNEZ, J. (Coord.) (2012). Agenda Digital para España. Informe de recomendaciones del Grupo de Expertos de alto nivel para la Agenda Digital para España. Madrid: Ministerio de Industria, Energía y Turismo. Disponible en: http://www.minetur.gob.es/telecomunicaciones/es-ES/Novedades/Docu ments/informe-recomendaciones-ade.pdf. (Consulta: 22.06.2012)

REBIUN, Red de Bibliotecas Universitarias. En: http://www.rebiun.org/index.html.

SHARMA, Divesh S. (2005). The association between ISO 9000 certification and financial performance. The International Journal of Accounting, 40, 151-172. DOI:10.1016/j.intacc.2005.01.011. 
SHORE, Cris (1993). Inventing the 'people's Europe': Critical approaches to European Community 'cultural policy'. San, New Series, 28(4), 779-800. Disponible en: http://www.jstor.org/stable/2803997 (24/04/2012).

YEUNG Mo-ching, Shirley (2011). Meeting customers' requirements with ISO audit quality management system (QMS) performance and organizational culture assessment. African Journal of Business Management, 5(9), 3497-3503. Disponible en: http://www.academicjournals.org/AJBM (Consulta: 20.03. 2012).

YORKE, Mantz (2000). Developing a quality culture in higher education. Tertiary Education and Management, 6, 19-36. Disponible en: $\mathrm{http} / / / \mathrm{www}$. springerlink.com/content/ qm8m3u40t6k70411/fulltext.pdf (Consulta: 10.04.2012)

ZOLTÁN Szabó, E.S.; Gábor, A. (2004). Simulating information policy making. Proceedings 16th European Simulation Symposium. György Lipovszki, István Molnár: SCS Press. ISBN 1-56555-286-5. 